\title{
Large Dermoid Cyst Presenting as Recurrent Pancreatitis
}

\author{
Emmanuel Oforia , Daryl Ramaia, b, c, Denzil Etienne ${ }^{\mathrm{a}}$, Madhavi Reddy ${ }^{\mathrm{a}}$, \\ Ghulamullah Shahzad ${ }^{\mathrm{a}}$
}

\begin{abstract}
Pancreatic dermoid cysts are rare and benign congenital abnormalities of germ cell origin. They occur equally in males and females without age predilection. Though radiographic imaging aids in the diagnosis, most times they are inconclusive and require fine-needle aspiration cytology for confirmation. We report the first case of a dermoid cyst presenting as recurrent pancreatitis in a 49-year-old male. Computed tomography scan, followed by magnetic resonance imaging, showed a complex cystic mass lesion adjacent to the pancreatic tail. Endoscopic ultrasound noted a large heterogeneous cystic lesion with hypoechoic wall layers in the tail of the pancreas without invading surrounding structures. Fine-needle aspiration cytology revealed numerous mature squamous cells consistent with a pancreatic dermoid cyst. We discuss the history, clinical presentation, diagnosis, and treatment of pancreatic dermoid cysts.
\end{abstract}

Keywords: Dermoid cyst; Pancreas; Pancreatitis; Endoscopic ultrasound; Fine-needle aspiration

\section{Introduction}

Dermoid cysts are rare congenital abnormalities of germ cell origin. Dermoid cysts of intrapancreatic accessory spleen have been reported in the literature; however, large dermoid cysts of the pancreas itself are very rare. Pancreatic dermoid cysts are benign and are usually detected as an incidental finding on abdominal imaging for other indications. To our knowledge, there are less than 40 reported cases of dermoid cyst originating in the pancreas. Herein, we report a case of a 49-year-old man with a history of pancreatitis who presented

Manuscript submitted June 16, 2017, accepted July 21, 2017

aDepartment of Gastroenterology and Hepatology, The Brooklyn Hospital Center, Academic Affiliate of The Icahn School of Medicine at Mount Sinai, Clinical Affiliate of The Mount Sinai Hospital, 121 Dekalb Avenue, Brooklyn, NY 11201, USA

bDepartment of Anatomical Sciences, St George's University School of Medicine, True Blue, Grenada, West Indies

${ }^{\mathrm{c} C}$ Corresponding Author: Daryl Ramai, Department of Anatomical Sciences, St George's University School of Medicine, True Blue, Grenada, West Indies. Email: dramai@sgu.edu

doi: https://doi.org/10.14740/gr876w with complaints of abdominal pain. He was found to have a large dermoid cyst which incited recurring symptoms of pancreatitis.

\section{Case Report}

A 49-year-old man with a past medical history of diabetes mellitus type 2, asthma, hypertension, hyperlipidemia, and pancreatitis 2 years prior, presented to the emergency department with complaints of abdominal pain for 2 days duration. Abdominal pain was in the epigastrium, and characterized as sharp, intermittent, and radiating to the left abdomen, back and groin. He reported an initial association with nausea and non-bilious, non-bloody vomiting. He denied associated fever, chills, weight loss, hematochezia, melena, or changes in bowel habits.

Physical examination revealed mild tenderness at the epigastrium without guarding or rebound tenderness. Laboratory findings included a white blood cell count of $6.2 \times 10^{3} /$ $\mu \mathrm{L}$ (normal range: $4.8-10.8 \times 10^{3} / \mu \mathrm{L}$ ), hemoglobin $13.4 \mathrm{~g} /$ dL (11.0 - $15.6 \mathrm{~g} / \mathrm{dL})$, hematocrit $41 \%$ (34-45\%), and platelet count $168 \times 10^{3} / \mu \mathrm{L}\left(130-400 \times 10^{3} / \mu \mathrm{L}\right)$. Blood urea nitrogen and creatinine were normal at $12 \mathrm{mg} / \mathrm{dL}(7-26 \mathrm{mg} / \mathrm{dL})$ and 1.1 $\mathrm{mg} / \mathrm{dL}(0.6-1.1 \mathrm{mg} / \mathrm{dL})$, respectively. Liver chemistries were normal. Serum lipase was elevated to $412 \mathrm{U} / \mathrm{L}$ (normal range). Serum triglyceride was $64 \mathrm{mg} / \mathrm{dL}$ (normal range). CEA and CA19-9 were also within normal limits.

Computed tomography (CT) scan of abdomen and pelvis showed an $8.2 \times 2.4 \times 3.6 \mathrm{~cm}$ fluid collection adjacent to the tail of the pancreas suspicious for a pseudocyst. Magnetic resonance imaging (MRI) showed a complex cystic mass lesion that could not be separated from the pancreatic tail. It demonstrated an irregular nodular wall and fluid component in the central portion measuring approximately $6.5 \mathrm{~cm}$ in the largest longitudinal diameter and $3 \mathrm{~cm}$ in the transverse diameter. The patient underwent an upper endoscopic ultrasound (EUS) with fine-needle aspiration (FNA). EUS noted a large heterogeneous cystic lesion with hypoechoic wall layers, which measured $56.8 \times 45.5 \mathrm{~mm}$ in the tail of the pancreas (Fig. 1). The lesion did not invade surrounding structures; however, a sluggish flow in the splenic vein was observed, likely due to splenic vein thrombosis. FNA revealed a muddy yellow fluid aspirate, drained until the cyst collapsed. Immunohistopathology revealed numerous mature squamous cells, morphologically consistent with a pancreatic dermoid cyst (Fig. 2). 


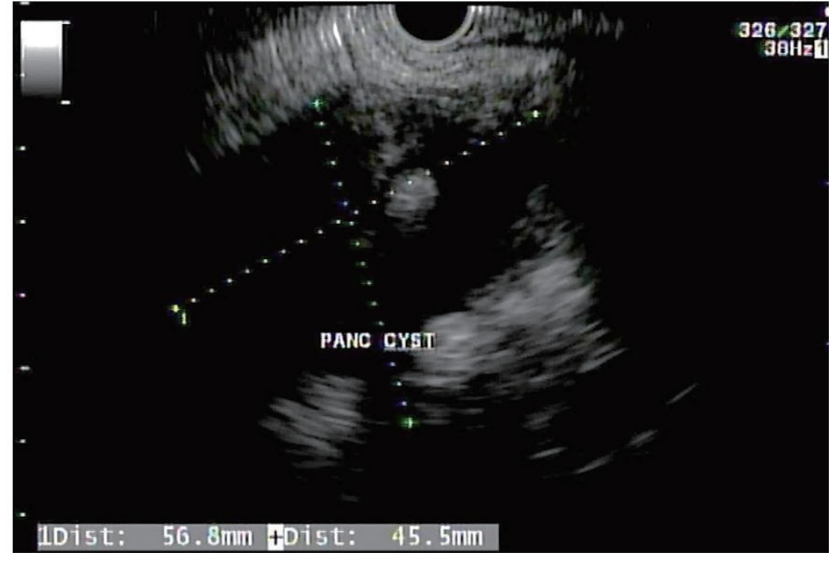

Figure 1. Endoscopic ultrasound showing a large heterogeneous cystic lesion with hypoechoic wall layers measuring $56.8 \times 45.5 \mathrm{~mm}$ in the tail of the pancreas.

\section{Discussion}

Dermoid cysts are mature cystic teratomas composed of tissue derived from all three germ layers [1]. They are divided into three classes, namely, 1) mature or benign, 2) immature or malignant, and 3) monodermal or highly specialized. Dermoid cysts, also known as mature teratomas, are mostly cystic with a predisposition toward ectodermal layers. Microscopically the cyst wall is lined by a single layer of keratinized stratified squamous epithelium with underlying connective tissue. In some instances, gross structures from ectodermal, endodermal, and mesodermal germ layers such as cartilage, bone, hair, teeth, sweat glands, thyroid tissue, etc. can be identified [2].

Pseudocysts are the most common cystic lesions of the pancreas and develop as a complication of acute pancreatitis. On the other hand, dermoid cysts are true cysts of the pancreas, constituting about $0.5 \%$ of all pancreatic cysts [3]. Anatomically, the pancreas is the rarest site of presentation, while the ovaries are the most common. However, these cysts have been reported in other areas of the body such as the testis, cranium, brain, mediastinum, omentum, retroperitoneum, and sacrococcygeal regions $[2,4]$. According to a review of published cases of pancreatic dermoid cysts, there is no gender or age predilection, and the most common presenting symptoms are abdominal or back pain [5]. Of these published cases, dermoid cysts were mostly found in the head and/or body of the pancreas, with only five cases involving the pancreatic tail [2, 4, 6-8].

Pancreatic dermoid cysts are diagnostically challenging. Most patients with pancreatic cysts are asymptomatic, and lesions are discovered incidentally upon imaging for other indications. Symptomatic patients complain of non-specific gastrointestinal symptoms such as abdominal pain, vomiting, nausea, weight loss, anorexia, fatigue, fever, and back pain [1, 2, 9]. CEA and CA19-9 serum markers are traditionally used to evaluate for neoplasms of the pancreas. In cases of dermoid cysts, these tumor markers are usually normal, as observed in our patient [2, 4]. However, one study reported persistent elevation of serum CA19-9 [10]. Another study found elevated CEA levels following aspiration of cyst contents, though no malignant cells were identified [11]. Thus, the use of CA19-9 and CEA has poor diagnostic utility in pancreatic dermoid cysts.

The radiologic appearance of pancreatic dermoid cysts depends on their composition. The more adipose tissue, fatfluid levels, and calcium contained within the cyst, the more

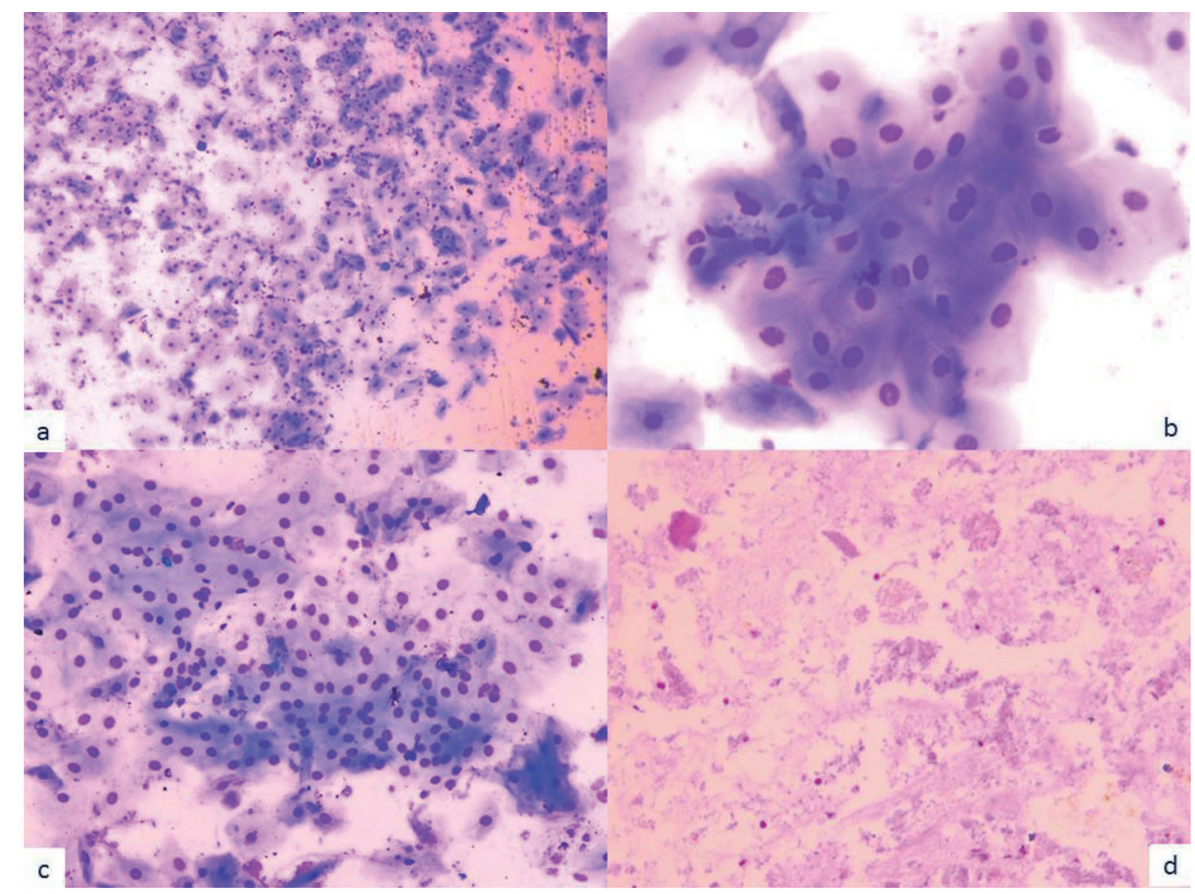

Figure 2. Microscopic examination shows mostly mature squamous cells in the background of acellular and degenerative material morphologically consistent with keratin debris (a: $\times 20$; $: \times 100 ; c: \times 40 ; d: \times 20$ ). 
indicative of a mature teratoma which is better detected by CT scan than ultrasonography $[12,13]$. On CT scan, adipose tissue appears hypoattenuated and calcifications are readily detected, which is highly suggestive of a dermoid cyst. However, on ultrasonogram, the fat within the cyst appears hyperechoic, and calcified areas are seen as areas of high intensity with acoustic shadowing $[9,13]$. The current recommendation requires use of FNA cytology to confirm the diagnosis of dermoid cyst [2].

While the malignant degeneration of dermoid cysts has never been reported, it is estimated that $7-10 \%$ of other retroperitoneal teratomas are malignant [14]. Furthermore, of all excised pancreatic cystic lesions, $70 \%$ have been reported as malignant or premalignant [15]. Thus, despite its benign nature, surgical resection remains the gold standard of treatment. Given the preoperative diagnostic challenge and history of recurrent pancreatitis symptoms, our patient was referred for surgical resection of the cystic lesion.

\section{Conclusion}

Pancreatic dermoid cysts are rare congenital abnormalities of germ cell origin. We report the first case of a dermoid cyst presenting as pancreatitis in a 49-year-old male. His clinical presentation and laboratory values were indicative of acute pancreatitis. Imaging, followed by FNA cytology, confirmed the presence of a large dermoid cyst of the pancreas. Heavy alcohol consumption and gallstones remain the leading cause of pancreatitis. However, when pancreatitis is suspected without any known etiology, clinicians should consider pancreatic dermoid cyst as part of the differential diagnosis.

\section{Conflicts of Interest}

None.

\section{References}

1. Seki M, Ninomiya E, Aruga A, Yamada K, Koga R, Saiura A, Yamamoto J, et al. Image-diagnostic features of mature cystic teratomas of the pancreas: report on two cases difficult to diagnose preoperatively. J Hepatobiliary Pancreat Surg. 2005;12(4):336-340.

2. Koomalsingh KJ, Fazylov R, Chorost MI, Horovitz J. Cystic teratoma of the pancreas: presentation, evaluation and management. JOP. 2006;7(6):643-646.

3. Farrell JJ. Prevalence, Diagnosis and management of pancreatic cystic neoplasms: current status and future directions. Gut Liver. 2015;9(5):571-589.

4. Tucci G, Muzi MG, Nigro C, Cadeddu F, Amabile D, Servadei F, Farinon AM. Dermoid cyst of the pancreas: presentation and management. World J Surg Oncol. 2007;5:85.

5. Lane J, Vance A, Finelli D, Williams G, Ravichandran P. Dermoid cyst of the pancreas: a case report with literature review. J Radiol Case Rep. 2012;6(12):17-25.

6. Judd ES. Cysts of the pancreas. Minnesota Medicine. $1921 ; 4: 75$.

7. Pomosov DV, Shpit'ko FS, Volianiuk MA. [Dermoid cyst of the pancreas in a child]. Vestn Khir Im I I Grek. 1973;110(4):92-93.

8. Das PC, Radhakrishna K, Rao PL. Cystic teratoma of the pancreas. Pediatr Surg Int. 1996;11(2-3):177-178.

9. Jacobs JE, Dinsmore BJ. Mature cystic teratoma of the pancreas: sonographic and CT findings. AJR Am J Roentgenol. 1993;160(3):523-524.

10. Micke O, Schafer U, Willich N. Persistent elevation of CA 19-9 levels in a patient with an extended retroperitoneal dermoid. Anticancer Res. 1999;19(4A):2717-2720.

11. Lee SE, Choi YS, Hong SU, Oh HC, Lee ES. Dermoid cyst of the pancreas: A rare cystic neoplasm. Int J Surg Case Rep. 2015;17:72-74.

12. Mester M, Trajber HJ, Compton CC, de Camargo Junior HS, de Almeida PC, Hoover HC, Jr. Cystic teratomas of the pancreas. Arch Surg. 1990;125(9):1215-1218.

13. Davidson AJ, Hartman DS, Goldman SM. Mature teratoma of the retroperitoneum: radiologic, pathologic, and clinical correlation. Radiology. 1989;172(2):421-425.

14. Albayrak A, Yildirim U, Aydin M. Dermoid cyst of the pancreas: a report of an unusual case and a review of the literature. Case Rep Pathol. 2013;2013:375193.

15. Zhang AY, Thompson SK, Game PA, Allin JJ. Images for surgeons. Cystic teratoma of the pancreas: a rare entity. ANZ J Surg. 2008;78(12):1130-1131. 